\title{
Coccygectomy; Is it Worthwhile in Every Patient with a Refractory Coccygodynia? An Updated Review
}

\author{
Farzad Omidi-Kashani $^{1 *}$, Ehsan Rafeemanesh ${ }^{2}$, Ali Ajvadi ${ }^{1}$, Ismaeil Garivani ${ }^{1}$ \\ ${ }^{I}$ Department of Orthopedic Surgery, Faculty of Medicine, Mashhad University of Medical Sciences, Mashhad, \\ Iran \\ ${ }^{2}$ Department of Occupational Medicine, Faculty of Medicine, Mashhad University of Medical Sciences, \\ Mashhad, Iran
}

*Corresponding Author: Farzad Omidi-Kashani, Orthopedic Department, Imam Reza Hospital, Imam Reza Square, Mashhad, Iran, Email: omidif@mums.ac.ir

\begin{abstract}
Coccyx is the last bone of the spine with wide variable attachment to the sacrum. This bone sometime becomes painful and causes a lot of disability. Coccydynia may be primary (de novo) or secondary to a pathology in another place. The most important way to differentiate primary from secondary coccydynia is local tenderness over the bone. The basis of treatment in coccydynia is conservative management and surgery is recommended only in patients with primary coccydynia in whom the pain does not respond to the less invasive therapeutic options. The usual surgical treatment for these patients is coccygectomy which if carefully performed, will have favorable clinical outcome. The most common and worst complications of this operation are infection and rectal injury, respectively.
\end{abstract}

Keywords: Coccygodynia; coccygectomy; Treatment.

\section{INTRODUCTION}

The coccyx is the last bone of the spinal column that is usually made of 3-5 separate or fused vertebrae attached to the sacrum. This attachment is variable and may be synovial, fibrocartilaginous, or ossified ${ }^{[1,2]}$. This bone is an important structure in the pelvis the serves as an attachment for ligamentous (sacrospinous, anterior, posterior coccygeal, and lateral sacrococcygeal ligaments) and muscular structures (external anal sphincter, levator ani, and coccygeus muscles). These components prevent the sagging of pelvic contents and are responsible for bowel continence ${ }^{[3]}$.

The name of the coccyx comes from cuckoo, a medium-sized slender bird with a curved beak. According to Postacchini and Massobrio, coccyx may have four types of configuration on lateral pain radiography ${ }^{[4]}$. In Type I, the coccyx is slightly curved forward with its apex facing downward, while in Type II this forward curvature is more dramatic and points straight forward. Type III, the coccyx acutely angles forward and in Type IV, the coccyx is subluxated at the sacrococcygeal or intercoccygeal joint.
Pain of the coccyx or coccygodynia is also known as coccydynia, coccalgia, or tailbone pain. The pain is not usually associated with a pain in the lumbar area and does not radiate or refer to the leg. Its prevalence is about $2.7 \%$ among the patients referring as low back pain (LBP) ${ }^{[5]}$. Although it can happen at any age, it usually begins around 40 with no ethnicity or race preference ${ }^{[6]}$. Coccyx is more prominent in female and may be due to this structural difference, the disease is much more common in female (female/male ratio of 5) [7]. Its predisposing factors include sacrococcygeal instability or fusion, high body mass index, trauma, childbirth, a history of surgery on lumbar spine or rectum, epidural injection, and inappropriate life style ${ }^{[6,8-11]}$. In this review, we focused on those patients in whom coccygectomy could yield a more satisfactory outcome.

\section{ETIOLOGY}

Coccydynia is a localized pain at the very bottom of the spine that usually gets worse with sitting or any activity that puts pressure on it. The most important issue in approaching a patient with coccydynia is distinction between the primary and secondary nature of the disease 
(Table 1). The most characteristic finding in primary versus secondary coccydynia is bone tenderness in palpation. In primary coccygodynia, the main pathology is coccyx itself. Two main causes for this condition are idiopathic and trauma ${ }^{[12]}$. Many different theories have been proposed for idiopathic type of coccygodynia including muscular spasm, functional neurosis, or psychiatric disturbance, but none of them has been proven ${ }^{[13]}$. In traumatic coccygodynia, the traumatic event may be acute (sudden fall from height), chronic (prolong sitting with bad posture especially on the hard surfaces), or acute on chronic (childbirth) ${ }^{[14,15]}$. The other less common causes of primary coccygodynia are listed in Table 1 [16-19].

In the secondary coccygodynia, the prime culprit is in another place and therefore in these cases, it is very important to identify the main pathology and not to convince the coccyx as the main offender. These types of coccydynia may arouse from the spinal column or internal viscerae (Table 1) ${ }^{[4,11,20,21]}$.

Table1. Underlying etiology of coccygodynia

\begin{tabular}{|c|c|}
\hline \multicolumn{2}{|r|}{ Etiology } \\
\hline Primary & $\begin{array}{ll}- & \text { Idiopathic, } \\
- & \text { Traumatic, } \\
- & \text { Levator ani syndrome, } \\
\text { - } & \text { Infection (osteomyelitis), } \\
\text { - } & \text { Tumor (intraossal lipoma, intraossal chondroma, precoccygeal cysts, sacrococcygeal } \\
\text { chordoma, giant cell tumor, intraductal schwannoma, perineural cyst, pilonidal cyst, } \\
\text { intraosseous lipoma, glomus tumor, sacrococcygeal meningeal cysts, and paragangliomas } \\
\text { of the caudal end of the os coccygis), } \\
\text { - } \quad \text { Avascular necrosis, } \\
-\quad \text { Sacrococcygeal osteoarthritis, } \\
-\quad \text { Soft tissues anomaly, } \\
\text { - } \quad \text { Bursitis, } \\
\text { - } \quad \text { Non-union. } \\
\text { - } \quad \text { Precongenital }\end{array}$ \\
\hline Secondary & $\begin{array}{l}\text { From the Spine: lumbar disc degeneration or herniation, lower lumbar spinal stenosis, } \\
\text { neural tumors (e.g., schwannoma, neurinoma, chordoma, arachnoid cysts of the cauda } \\
\text { equina), lumbosacral injuries. } \\
\text { 2- From the viscerae: Infections and tumors of the rectum, sigmoid colon, and urogenital } \\
\text { system, metastases. }\end{array}$ \\
\hline
\end{tabular}

\section{Clinical Presentation}

Most of the patients have a history of a trauma to the coccyx. This trauma may be due to an acute event (falling from the height or direct kick to the coccyx) or chronic poor posture while sitting. Patient usually complain of a pain that is localized to the bottom of the spine. The pain commonly alleviates with standing and walking but aggravates by sitting. Some of the patients complain of the pain in lying position, during defecation, sexual activity, and riding a bicycle or a motorbike ${ }^{[21,22]}$.

\section{Diagnostic Tests}

The correct diagnosis needs a complete medical history and appropriate physical examination. History taking should include information about the nature of the pain, chronicity of the pain, the presence of an initial traumatic event, severity and course of the complains, bowel habit, and existence of associated red flags (night pain, unwanted weight loss, paresthesia, weakness, sphincter disturbance, etcetera). Paying attention to the past and family medical history, lifestyle habits is also very important.

Physical examination for coccydynia comprises of three parts. First, observation for rolling out skin rash, fissure, ulcer, tumefaction, etcetera. Second, palpation for checking local tenderness, swelling, bony spur, cyst, and so on. Third, intrarectal manipulation to assess coccygeal mobility and muscular tension. Note that local tenderness is the best clinical indicator of primary versus secondary coccydynia ${ }^{[23]}$.

Diagnostic paraclinical tests are only indicated in refractory or unusual cases. These tests include:

\subsection{Plain Radiography}

Anteroposterior and lateral views of the sacrococcygeal area may be helpful in revealing fractures, dislocations, osteophytes, and abnormal sacrococcygeal curvature but lateral dynamic views in standing and sitting positions 
can detect changes in coccygeal alignment more clearly. Standing lateral view is taken when the patient has maintained this position for more than 10 minutes. In sitting view, the patient is asked to sit while the back is straight and the thighs are level. Then the patient should bend backward until the pain is reproduced and at this point, the lateral view is taken ${ }^{[24]}$. Normal range is less than $25 \%$ translation of the coccyx and a rage of 5 to 25 degrees of rotation. Fogel et al. found that too much mobility or too much stiffness are both pathologic and can be detected in as much as $70 \%$ of the patients with coccydynia ${ }^{[25]}$. Intercoccygeal angle (the angle between the midline of the first and last coccygeal segment) is a useful parameter in assessing forward angulation of the coccyx. Kim and Suk proposed that this angle is greater in idiopathic versus traumatic cases ${ }^{[26]}$.

\subsection{Local Injection}

Direct introducing a needle to the point of maximum tenderness to elicit the same pain as previously felt by the patient and subsequent pain relief by injecting a local anesthetic agent may be helpful in diagnosis and treatment of the disease ${ }^{[23]}$

\subsection{Discography}

Coccygeal discography may be helpful in detecting the precise location of the pain, although this is not universally accepted. Maigne et al. reported this technique was successful in 44 of 55 cases with coccygodynia especially in cases with subluxation or hypermobility ${ }^{[27]}$.

\subsection{Whole Body Bone Scan, CT or MRI Scanning}

These modalities are especially helpful in patients suspicious for a fracture, tumor, or infection. MRI should exclude any simultaneous pathology in spinal cord, intervertebral disc, or spinal canal (like lumbar spinal stenosis) ${ }^{[15,23,}$ 28].

\section{Conservative Treatment}

Although several therapeutic options have been proposed for the patients with coccygodynia, non-operative treatment continues to be basis for the disease. Nonsteroidal anti-inflammatory drugs (NSAIDs) to lessen the pain associated with inflammation are usually the first step of treatment. Laxatives are prescribed to reduce pressure on the coccyx. Modification in life style in order to avoid excessive pressure on the tailbone in sitting position (using soft seats, ring-shaped cushions, and etcetera) is another mandatory principle in conservative treatment.
Diagnostic and therapeutic injection (at the point of maximum tenderness) is universally recommended ${ }^{[19]}$. This can be performed by local anesthetic agent and steroids. Khan et al. suggested dextrose prolotherapy before undergoing coccygectomy, as an effective therapeutic option in the patients with chronic, refractory coccygodynia, although there is still not a randomized study to compare prolotherapy with local steroid injection ${ }^{[29]}$. Ganglion impar injection (block) was recommended by some authors ${ }^{[30-32]}$. This ganglion is also known as ganglion of Walther (or azygos) and is the terminal convergence of two sympathetic chains in front of the coccyx ${ }^{[33]}$. It was proposed that even a single local nerve block injection at this area under fluoroscopic control might create complete pain relief in the cases with refractory coccygodynia ${ }^{[30]}$. This injection was also proposed in other patients with chronic pelvic pain due to cancer or so on ${ }^{[32,34]}$.

Another therapeutic option is applying a kind of skin patches. Levesque et al. proposed highconcentration capsaicin patch in the management of coccydynia and reported great success ${ }^{[35]}$. Ergonomic adaptation (buttock strapping, postural training, firm corset, and etcetera) and coccygeal massage or manipulation are also recommended ${ }^{[8,11,19,36]}$. Thiele massage was invented to relieve the spastic levator ani and coccygeus on both sides [37]. In cases with coccygeal mobility, digital manipulation may be more helpful ${ }^{[36,38]}$. Other less common modalities sometimes mentioned include radiofrequency ablation and extracorporeal shock wave therapy (ECSWT) [39-41]

\section{Operative TreatMent}

In those patients with primary coccygodynia in whom aggressive conservative treatment does not yield a satisfactory outcome and the disease causes a problem in activity of daily living, surgical intervention as the last resort may become necessary. This operation is contraindicated in the patients with secondary coccygodynia or local infection. This procedure can be performed as partial or complete coccygectomy but complete coccygectomy has a lower incidence of failure ${ }^{[42]}$.

Usually a low residue diet is recommended 1 to 5 days before surgery. Coccygectomy can be performed by two surgical (Powers and Gardner) techniques ${ }^{[43,}{ }^{44]}$.In both techniques, the patient is placed in a prone position, hips in flexion and the buttocks separated by a hard 
tape. In Powers technique, a longitudinal incision is made from the sacrococcygeal joint to the tip of the coccyx. The bone is excised subperiosteally and distal end of the sacrum beveled. In Gardner technique, the tip of the coccyx is first elevated by blunt dissection then sharply separated from the external anal sphincter. By a moist sponge, the coccyx is bluntly mobilized up to the sacrococcygeal joint and then the coccyx sharply dissected here.

Coccygectomy can be performed subperiosteally or by its periosteal sleeve. Periosteal preservation has been shown to be associated with faster recovery and less postoperative infection (may be due to less probability of rectal injury). After coccygectomy, distal prominent end of sacrum is beveled and dead space should be eliminated by suturing the aponeurotic tissues. Any persistence of dead space allows hematoma formation and subsequent infection.

\section{COMPLiCATIONS}

The operated patients should be informed that postoperative improvement in symptoms is usually gradual and may be observed after a few months. An overall complication rate of $11 \%$ was reported in the literature ${ }^{[45]}$. Needless to say that due to the proximity of the surgical site to the anus, the most common complication of this procedure is infection. Infection rate has been reported to be occurred as high as 30\% and usually caused by gram-negative rods ${ }^{[46]}$. In order to reduce this complication, some authors proposed a preoperative rectal enema, a particular topical wound adhesive, closure of the incision in two layer, and postoperative antibiotic therapy with second-generation cephalosporins for 5 days ${ }^{[47,48]}$.

The worst complication is rectal injury as the dissection goes away from the subperiosteal region [10]. Injury to the external anal sphincter can create bowel incontinence. Ganglion impar is the terminal ganglion of the paravertebral sympathetic nervous system and closely located in anterior surface of the coccyx. Damage to this ganglion may interfere with sympathetic nerve function. Other complications occasionally quoted include hematoma, wound healing problems like wound dehiscence and delayed healing.

\section{CONClusion}

The basis for treatment of coccydynia is conservative but persistent cases are medical challenges. Coccygectomy is not suitable for every patient with recalcitrant coccydynia and may create catastrophic outcomes. In order to decide on surgery, the treating surgeon by taking a history, clinical examination, and if necessary, paraclinical measures, should first distinguish between primary and secondary illness. Coccygectomy is only appropriate for those patients with primary coccydynia in whom the pain does not respond to the less invasive therapeutic options. The most common and worst complications of this operation are infection and rectal injury, respectively.

\section{COMPETING INTERESTS}

The authors have no competing interests. No benefits in any form have been received or will be received from a commercial party related directly or indirectly to the subject of this article.

\section{ACKNOWLEDGEMENT}

The authors thank Dr Mohammad Hosein Ebahimzadeh, the head of the Orthopedic Research Center, Mashhad University of Medical Sciences, for giving us the facilities of the center.

\section{REFERENCES}

[1] Jason T. K. Woon, Vivekanandan Perumal, Jean-Yves Maigne, Mark D. Stringer. CT morphology and morphometry of the normal adult coccyx. Eur Spine J. 2013; 22(4): 863-70.

[2] Saluja P G. The incidence of ossification of the sacrococcygeal joint. J Anat. 1988; 156: 11-5.

[3] Woon JT, Stringer MD. Clinical anatomy of the coccyx: A systematic review. Clin Anat. 2012; 25(2): 158-67.

[4] Postacchini F, Massobrio M. Idiopathic coccygodynia. Analysis of fifty-one operative cases and a radiographic study of the normal coccyx. J Bone Joint Surg Am. 1983; 65(8): 1116-24.

[5] Ghormley RK. An Etiologic Study of Back Pain. Radiology. 1958; 70(5): 649-53.

[6] Patel R, Appannagari A, Whang PG. Coccydynia. Curr Rev Musculoskelet Med. 2008; 1(3-4): 223-6.

[7] Wray CC, Easom S, Hoskinson J. Coccydynia. Aetiology and treatment. J Bone Joint Surg Br. 1991; 73(2): 335-8.

[8] Howard PD, Dolan AN, Falco AN, Holland BM, Wilkinson CF, Zink AM. A comparison of conservative interventions and their effectiveness for coccydynia: a systematic review. J Man Manip Ther 2013; 21(4): 213-9.

[9] Kwon HD, Schrot RJ, Kerr EE, Kim KD. Coccygodynia and coccygectomy. Korean J Spine. 2012; 9(4):326-33. 
[10] Karadimas EJ, Trypsiannis G, Giannoudis PV. Surgical treatment of coccygodynia: an analytic review of the literature. Eur Spine J. 2011; 20(5): 698-705.

[11] Nathan ST, Fisher BE, Roberts CS. Coccydynia: a review of pathoanatomy, aetiology, treatment and outcome. J Bone Joint Surg Br. 2010; 92(12): 1622-7.

[12] Grgić V. Coccygodynia: etiology, pathogenesis, clinical characteristics, diagnosis and therapy. Lijec Vjesn 2012; 134(1-2): 49-55.

[13] Suduca P. Anorectal neuralgias. Ann Gastroenterol Hepatol (Paris). 1985; 21(6): 393-6.

[14] Ramieri A, Domenicucci M, Cellocco P, Miscusi M, Costanzo G. Acute traumatic instability of the coccyx: results in 28 consecutive coccygectomies. Eur Spine J. 2013; 22 Suppl 6: S939-44

[15] Maigne JY, Pigeau I, Aguer N, Doursounian L, Chatellier G. Chronic coccydynia in adolescents. A series of 53 patients. Eur J Phys Rehabil Med. 2011; 47(2): 245-51.

[16] Albrecht S, Hicks MJ, Antalffy B. Intracoccygeal glomus bodies and their relationship to coccygodynia. Surgery 1994; 115(1): 1-6.

[17] Duncan L, Halverson J, DeSchryverKecskemeti K. Glomus tumor of the coccyx. A curable cause of coccygodynia. Arch Pathol Lab Med. 1991; 115(1): 78-80.

[18] Moon SG, Kim NR, Choi JW, Yi JG. Acute coccydynia related to precoccygeal calcific tendinitis. Skeletal Radiol. 2012; 41(4): 473-6.

[19] Lirette LS, Chaiban G, Tolba R, Eissa H. Coccydynia: an overview of the anatomy, etiology, and treatment of coccyx pain. Ochsner J. 2014; 14(1): 84-7.

[20] Cockbain AJ, Morrison CP, Davies JB. Coccydynia secondary to a large pelvic tumor of anorectal origin. Spine J. 2011; 11(7): 683.

[21] Blocker O, Hill S, Woodacre T. Persistent coccydynia--the importance of a differential diagnosis. BMJ Case Rep. 2011; 2011. pii: bcr0620114408.

[22] Andromanakos NP, Kouraklis G, Alkiviadis K. Chronic perineal pain: current pathophysiological aspects, diagnostic approaches and treatment. Eur J Gastroenterol Hepatol. 2011; 23(1): 2-7.

[23] Foye PM. Coccydynia: Tailbone Pain. Phys Med Rehabil Clin N Am. 2017; 28(3): 539-49.

[24] Maigne JY, Lagauche D, Doursounian L. Instability of the coccyx in coccydynia. J Bone Joint Surg Br. 2000; 82(7): 1038-41.

[25] Fogel GR, Cunningham PY 3rd, Esses SI. Coccygodynia: evaluation and management. J Am Acad Orthop Surg. 2004; 12(1):49-54.
[26] Kim NH, Suk KS. Clinical and radiologic differences between traumatic and idiopathic coccygodynia. Yonesi Med J. 1999; 40(3): 21520.

[27] Maigne JY, Guedj S, Straus C. Idiopathic coccygodynia. Lateral roentgenograms in the sitting position and coccygeal discography. Spine 1994; 19(8): 930-4.

[28] Trouvin AP. Reply to the comment of Foye et al. "MRI, CT scan, and dynamic radiographs for coccydynia". Joint Bone Spine. 2014; 81(3): 280.

[29] Khan SA, Kumar A, Varshney MK, Trikha V, Yadav CS. Dextrose Prolotherapy for recalcitrant coccydynia. J Orthop Surg (Hong Kong). 2008; 16(1): 27-9.

[30] Foye PM, Buttaci CJ, Stitik TP, Yonclas PP. Successful injection for coccyx pain. Am J Phys Med Rehabil. 2006; 85(9): 783-4.

[31] Bogduk N. Ganglion Impar Blocks for Coccydynia: A Case Series Prerequisite for Efficacy Trial. Pain Med. 2015; 16(7): 1245.

[32] Walters A, Muhleman M, Osiro S, et al. One is the loneliest number: a review of the ganglion impar and its relation to pelvic pain syndromes. Clin Anat. 2013; 26(7): 855-61.

[33] Scott-Warren JT, Hill V, Rajasekaran A. Ganglion impar blockade: a review. Curr Pain Headache Rep. 2013; 17(1): 306.

[34] Malec-Milewska M, Horosz B, Kolęda I, Sękowska A, Kucia H, Kosson D, Jakiel G. Neurolytic block of ganglion of Walther for the management of chronic pelvic pain. Wideochir Inne Tech Maloinwazyjne. 2014; 9(3): 458-62.

[35] Levesque A, Riant T, Labat JJ, Ploteau S. Use of high-concentration capsaicin patch for the treatment of pelvic pain: observational study of 60 inpatients. Pain Physician. 2017; 20(1): E161-7.

[36] Emerson SS, Speece AJ 3rd. Manipulation of the coccyx with anesthesia for the management of coccydynia. J Am Osteopath Assoc. 2012; 112(12): 805-7.

[37] Montenegro ML, Mateus-Vasconcelos EC, Candido dos Reis FJ, Rosa e Silva JC, Nogueira AA, Poli Neto OB. Thiele massage as a therapeutic option for women with chronic pelvic pain caused by tenderness of pelvic floor muscles. J Eval Clin Pract. 2010; 16(5): 981-2.

[38] Maigne JY, Chatellier G, Faou ML, Archambeu M. The treatment of chronic coccydynia with intrarectal manipulation: a randomized controlled study. Spine. 2006; 31(18): E621-7.

[39] Gopal H, Mc Crory C. Coccygodynia treated by pulsed radio frequency treatment to the ganglion of Impar: A case series. J Back Musculoskelet Rehabil. 2014; 27(3) 349-54.

[40] Marwan Y, Dahrab B, Esmaeel A, Ibrahim SA, Al-Failakawi J. Extracorporeal Shock wave 
therapy for the treatment of coccydynia: a series of 23 cases. Eur J Orthop Surg Traumatol 2017; 27(5): 591-8.

[41] Chen Y, Huang-Lionnet JHY, Cohen SP. Radiofrequency Ablation in Coccydynia: A Case Series and Comprehensive, EvidenceBased Review. Pain Med. 2017; 18(6): 111130.

[42] Trollegaard AM, Aarby NS, Hellberg S. Coccygectomy: an effective treatment option for chronic coccydynia: retrospective results in 41 consecutive patients. J Bone Joint Surg Br. 2010; 92(2): 242-5.

[43] Powers JA. Coccygectomy. South Med J. 1957; 50(5): 675-8.

[44] Gardner RC. An improved technic of coccygectomy. Clin Orthop Relat Res. 1972. 85: 143-5.
[45] Antoniadis A, Ulrich NH, Senyurt $\mathrm{H}$. Coccygectomy as a surgical option in the treatment of chronic traumatic coccygodynia: a single-center experience and literature review. Asian Spine J. 2014; 8(6): 705-10.

[46] Bayne O, Bateman JE, Cameron HU. The influence of etiology on the results of coccygectomy. Clin Orthop Relat Res. 1984; 190: 266-72.

[47] Hodges SD, Eck JC, Humphreys SC. A treatment and outcomes analysis of patients with coccydynia. Spine J. 2004; 4(2): 138-40.

[48] Doursounian L, Maigne JY, Cherrier B, Pacanowski J. Prevention of postcoccygectomy infection in a series of 136 coccygectomies. Int Orthop. 2011; 35(6): 87781 .

Citation: Farzad Omidi-Kashani, Ehsan Rafeemanesh, Ali Ajvadil, Ismaei Garivani. Coccygectomy; Is it Worthwhile in Every Patient with a Refractory Coccygodynia? An Updated Review. ARC Journal of Orthopedics. 2017; 2(1):18-23. doi:dx.doi.org/ 10.20431/2456-0588.0201003.

Copyright: (C) 2017 Authors. This is an open-access article distributed under the terms of the Creative Commons Attribution License, which permits unrestricted use, distribution, and reproduction in any medium, provided the original author and source are credited. 